\title{
Management Of Muscle Relaxant In A Patient With Bulbar Symptoms And Advanced Inferior Motor Neuron Disease.
}

\author{
Aragón Benedí, Cristian; Visiedo Sanchez, Sara; \\ Pascual Bellosta, Ana; Ortega Lucea, Sonia; Martínez Ubieto, Javier; Gil Bona, Jesús; \\ Department Of Anesthesiology And Critical Care, Hospital Miguel Servet, Zaragoza, Spain.
}

\section{Introduction}

Lower motor neuron diseases are characterized by muscle weakness and progressive paralysis that often results in death due to respiratory failure and hypoventilation in one to three years. In other cases, the onset is marked clinically due to the lesion of the bulbar nerves with dysarthria, dysphagia and dyspnea.

In these patients, general anesthesia with endotracheal intubation can ensure adequate intraoperative ventilation, however respiratory difficulties, abnormal response to muscle relaxants, higher risk of aspiration, airway obstruction and regurgitation may result in prolonged mechanical ventilation in the postoperative period.

The objective of this work is to present a case of general anesthesia in a patient with lower motor neuron disease reviewing the latest evidence of the management of neuromuscular blockade in these pathologies.

\section{Clinical case.}

A clinical case of a wowan of 45 -year-old $(70 \mathrm{~kg})$, allergic to piroxicam in treatment with Prednisone, with a history of dyslipidemia, migraine with aura, Cushing's disease and degenerative lower motor neuron disease.

The patient presented progressive distal musculature weakness in inferior limbs and right arm, as well as bulbar symptoms. She presented swallowing disorder, odynophagia for solids with weight loss in the last year of $25 \mathrm{~kg}$, dysphonia and fluctuating stridor. Bilateral paralysis of vocal cords in adduction was seen in fibrolaryngoscopy by the ENT, being candidate for laryngeal microsurgery for control of her bulbar symptomatology. The patient presented high risk in the airway management so she was informed the possibility of tracheostomy being accepted by the patient.

Anesthesia was induced with fentanyl $100 \mu \mathrm{g}$, propofol $170 \mathrm{mg}$ and rocuronium $30 \mathrm{mg}$ and maintained with sevorane $\mathrm{I}-2 \%$, and continuous perfusion of remifentanil $0.05 \mu \mathrm{g} / \mathrm{kg} / \mathrm{min}$. Intubation was performed on the first attempt with direct laryngoscopy with a 5.5 metal tube. The neuromuscular response was monitored by train of four (TOF) during all the intervention. A new dose of rocuronium 10 mg after 30 minutes of induction was needed. A partial cordotomy was performed in I/3 posterior with CO2 laser. After one hour value of TOF ratio was $99 \%$ and was not required reversal of neuromuscular blockade. Awakening and extubation was performed without complications.

After completing the surgery the patient was transferred to the critical care unit (ICU) for continuos neuromuscular monitoring by TOF. The patient did not have residual muscle blockade, weakness, stridor or respiratory clinic, and twenty four hours later the patient was discharge without complications to the ward managed by the ENT Service.

\section{Discussion.}

These patients with motor neuron diseases are hypersensitive to non-depolarizing neuromuscular blockers. However, rocuronium has been demonstrated to be safe due to its rapid onset of action and the possibility of antagonizing the blockade with Sugammadex. Depolarizing relaxants are extremely contraindicated in these patients due to they may cause myotonic reactions and massive hyperkalemia.

The use of other non-neuromuscular relaxants is not recommended due to the risk of residual postoperative blockade. Reversal with cholinesterase inhibitors is not indicated due to the unpredictable effect and they may produced unwanted cholinergic effects that can lead to respiratory complications. In addition to a correct reversal of the blockade, an objective and quantitative neuromuscular monitoring is essential in order to control the depth of neuromuscular relaxation during and at the end of the surgery.

Propofol and remifentanil are agents with minimal interaction with neuromuscular function, so the recovery of these agents is rapid, even in patients with neuromuscular disorders. In some patients with more advanced clinics, the application of BIPAP ventilation is recommended in the immediate postoperative period since it is demonstrated to improve survival in these motor neuron desease.

I. Kowalik MM, Smiatacz T, Pajuro R, et al.Anaesthesia for ophthalmologic surgical procedures in a patient with advanced amyotrophic lateral sclerosis: a case report. Cases J. 2008; I(I):338.

2. Díaz A, Ledesma B, Gilsanz F. [Thoracic paravertebral block plus pectoral nerve block for mastectomy in a patient with advanced amyotrophic lateralsclerosis]. Rev Esp Anestesiol Reanim. 20I 4;6I (3): I 72-3.

3. Stewart PA, Phillips S, De boer HD. Sugammadex reversal of rocuronium-induced neuromuscular blockade in two types of neuromuscular disorders: Myotonic dystrophy and spinal muscular atrophy. Rev Esp 